\title{
Criteria for the selection of switch OTC drugs based on patient benefits, efficacy, and safety [II]: Comparing the physicochemical and pharmaceutical properties of brand-name and switch OTC terbinafine hydrochloride cream
}

\author{
Motoari Takata ${ }^{1}$, Yuko Wada ${ }^{2}$, Yurika Iwasawa ${ }^{3}$, Miyuki Kumazawa ${ }^{4}$, Ken-ichi Shimokawa ${ }^{3}$, \\ Fumiyoshi Ishii',** \\ ${ }^{1}$ Welcia Yakkyoku Co. Ltd., Tokyo, Japan; \\ ${ }^{2}$ Department of Self-medication and Health Care Sciences, Meiji Pharmaceutical University, Tokyo, Japan; \\ ${ }^{3}$ Department of Pharmaceutical Sciences, Meiji Pharmaceutical University, Tokyo, Japan; \\ ${ }^{4}$ Department of Mathematical Science, Meiji Pharmaceutical University, Tokyo, Japan.
}

\begin{abstract}
Summary The physicochemical properties $(\mathrm{pH}$, yield value, and squeeze force) of a drug for dermatomycosis, a terbinafine hydrochloride-containing cream (a brand-name product), and 12 over-the-counter drugs (OTCs) were measured and compared to ascertain the characteristics of each product. The $\mathrm{pH}$ of the brand-name product, Lamisil, was 4.1, and that of the OTC products ranged from 4.2 to 7.6; Lamisil Plus (7.6) had a significantly higher $\mathrm{pH}$. Moreover, the yield value for Lamisil, as an index of cream ductility, was $\mathbf{1 2 8}$ dyn $/ \mathrm{cm}^{2}$, and that for the OTC products ranged from 110 to $887 \mathrm{dyn} / \mathrm{cm}^{2}$. In particular, the OTC products Damalin $\left(887 \mathrm{dyn} / \mathrm{cm}^{2}\right)$, Barriact $\left(512 \mathrm{dyn} / \mathrm{cm}^{2}\right)$, and Exiv Deep $(663 \mathrm{dyn} /$ $\mathrm{cm}^{2}$ ) had a significantly higher yield value. In addition, the squeeze force was measured by attaching a $\mathrm{HapLog}^{\circledR}$ to the thumb and second finger. The squeeze force for Lamisil was 12.9 $\mathrm{N}$, and that for the OTC products ranged from 1.8 to 14.6 N. The OTC product Bilumon (1.8 N) had a significantly lower squeeze force. These results indicated that there were marked differences in the pharmaceutical properties of brand-name and OTC products. External preparations are characterized by their feel during use. Based on the current results, the pharmaceutical characteristics of drugs resulted in differences in their feel during use, suggesting that products appropriate for individual patients can be recommended.
\end{abstract}

Keywords: Cream, terbinafine hydrochloride, brand-name drug, OTC, HapLog ${ }^{\circledR}$

\section{Introduction}

In Japan, the continued increase in national healthcare expenditures poses a serious social problem (1). One way to reduce medical expenditures is by promoting the use of over-the-counter (OTC) drugs in self-medication (2). Since January 1, 2017, if a person buys a "switch OTC" (an OTC drug switched from a prescription drug), the cost of the drug can be deducted from the person's income via an exemption for healthcare costs

*Address correspondence to:

Dr. Fumiyoshi Ishii, Department of Self-medication and Health Care Sciences, Meiji Pharmaceutical University, 2-522-1, Noshio, Kiyose, Tokyo 204-8588, Japan.

E-mail: fishii@my-pharm.ac.jp under the system for taxation of self-medication as part of an effort to maintain and promote health and disease prevention (2). This reflects the government's policy to promote self-medication and encourage a shift from drugs that require a prescription to OTC drugs that require no prescription ("Patients should be responsible for their own health and treat mild physical ailments themselves (3)") (4).

Pharmacists can contribute greatly to the promotion of self-medication by sufficiently understanding the equivalence of OTC drugs to brand-name drugs and their physicochemical characteristics and encouraging their use with appropriate evaluation. However, there are no clear criteria for the selection of OTC drugs, so drugs cannot be easily selected to suit individual patients. 
The current authors previously reported that various patient needs could be met by ascertaining the physicochemical and pharmaceutical properties of various dosage forms, most of which are external preparations that markedly differ in their feel during use: ointments, creams, lotions (5-7), ophthalmic liquids/ solutions (8), nasal sprays (9), and tapes $(10,11)$.

The aim of the current study was to compile information to help pharmacists select appropriate preparations according to patient needs by comparing and assessing pharmaceutical characteristics of a brandname drug and OTC products. The brand-name and OTC drugs were creams containing terbinafine hydrochloride, which is a treatment for dermatomycosis.

\section{Materials and Methods}

\subsection{Materials}

Pharmaceutical creams containing terbinafine hydrochloride (1 brand-name "Lamisil ${ }^{\circledR}$ cream $1 \%$ " and 12 OTCs) were used in this study. The product name, abbreviated name, class, company name, lot number, and container of these products are listed in Table 1. In addition, OTC products were specifically those on the market (in drugstores, on the Internet, etc.) among all products registered with the Pharmaceutical and Medical Devices Agency (PMDA) (12).

\subsection{Measurement of $p H$}

The $\mathrm{pH}$ of each cream was measured using the HI 99161N pH meter (Hanna Instruments Japan Co. Ltd., Chiba, Japan) for dairy products and semi-solid foods. Measurement was performed 10 times at $24 \pm 2{ }^{\circ} \mathrm{C}$, and the mean \pm standard deviation (S.D.) was calculated.

\subsection{Measurement of ductility}

The spread diameter of each cream was measured using the 419 spreadmeter (Rigo Co., Ltd., Tokyo,
Japan). Measurement was performed 8 times at room temperature $\left(24 \pm 2^{\circ} \mathrm{C}\right)$, and the mean value was calculated. The spread diameter was determined at 16 points between 5 and 1,800 seconds after the start of measurement. A semilog graph was prepared by plotting the spread diameter $(\mathrm{cm})$ of each cream on the longitudinal axis and plotting the time (seconds) needed for the cream to spread on the transverse axis. The spread and viscosity of each cream were calculated from the slope and y-intercept $(13,14)$. Moreover, the yield value was calculated using a previously reported formula (15).

\subsection{Measurement of squeeze force}

To assess the squeeze force with which a preparation was expelled from a container, a wearable tactile sensor (Haptic Skill Logger (HapLog ${ }^{\circledR}$ ), Kato Tech Co., Ltd., Kyoto, Japan) was used to evaluate the sense of touch $(16,17)$. Tactile sensors were attached to the right thumb and second finger, and the total force (thumb + second finger) required to squeeze 1 fingertip unit (FTU: volume of cream squeezed between the first joint and tip of the second finger) of cream while placing the center of the container between the thumb and second finger was regarded as the squeeze force $(\mathrm{N})$. These sensors facilitate the simultaneous assessment of the finger contact force and sense of touch of the person wearing the sensor through the free sense of touch at the fingertip, but there are errors in the contact force due to individual differences in the method of attachment or size of the finger. This is why the values were corrected for each person. Measurement was performed 7 times per container of cream with the same lot number. In addition, measurement was performed for a total of 3 bottles if a product had different lot numbers. The mean of the respective means was then calculated to serve as the value for the product.

\subsection{Statistical analysis}

Values were compared using Dunnett's test (18). A $p$

Table 1. Creams used in this experiment

\begin{tabular}{|c|c|c|c|c|c|}
\hline Product name & Abbreviated name & Class & Company & Lot number & Container \\
\hline Lamisil $^{\circledR}$ Cream 1\% & Lamisil & Brand-name & Novartis Pharma K. K. & P0942 & AT \\
\hline Damalin Grande X & Damalin & OTC & Taisho Pharm. Co., Ltd. & 024P1 & AT \\
\hline Barriact Hi Cream & Barriact & OTC & Zeria Pharm. Co., Ltd. & SZ01 & AT \\
\hline Salirabate EX Cream & Salirabate & OTC & Japan Medic Co., Ltd. & 14013 & AT \\
\hline Mentholatum Exiv Cream E & Exiv & OTC & Rohto Pharm. Co., Ltd. & 4E4 & LT \\
\hline Mentholatum Exiv Deep 10 Cream & Exiv Deep & OTC & Rohto Pharm. Co., Ltd. & 4D3 & PB \\
\hline Terubine EX Cream & Terubine & OTC & Chugai Iyaku Seisan Co., Ltd. & 4202 & AT \\
\hline Next Cream 24 & Next & OTC & Shinsei Yakuhin Co., Ltd. & $4 \mathrm{~T} 1$ & AT \\
\hline Bilumon TF Cream EX & Bilumon & OTC & Shinshin Pharm. Co., Ltd. & E301 & LT \\
\hline Mailuzon TL Cream & Mailuzon & OTC & Fuji Pharma Co., Ltd. & 30101 & AT \\
\hline Lamisil AT Cream & Lamisil AT & OTC & Novartis Pharma K. K. & P0121 & $\mathrm{AT}$ \\
\hline Lamisil Plus Cream & Lamisil Plus & OTC & Novartis Pharma K. K. & 4B02 & AT \\
\hline Lamisil Cure Gel & Lamisil Gel & OTC & Novartis Pharma K. K. & $4 \mathrm{~A} 01$ & AT \\
\hline
\end{tabular}

AT: Aluminum tube, LT: Laminated tube, PB: Plastic bottle. 
value of 0.05 or 0.01 was considered significant.

\section{Results}

\subsection{Measurement of $p H$}

Previous studies have indicated that the $\mathrm{pH}$ influences the stability of drugs. The $\mathrm{pH}$ of each preparation used in this experiment was measured, and the results are shown in Figure 1. The $\mathrm{pH}$ of the brand-name drug, Lamisil ( $\mathrm{pH} 4.1$ ), and that of the OTC products ranged from 4.2 to 7.6 (Figure 1). In particular, the OTC products Exiv Deep ( $\mathrm{pH}$ 6.9), Next (pH 6.9), Lamisil Plus (pH 7.6), and Lamisil Gel (pH 6.7) had a significantly higher $\mathrm{pH}$.

\subsection{Measurement of ductility}

When a cream is applied to the skin, its feel depends on its ductility and viscosity. The spread diameter and time needed for each cream to spread were measured using a spreadmeter. In addition, a semilog graph (longitudinal axis: spread diameter, transverse axis: time needed to spread) was prepared to calculate the slope and $y$-intercept for each preparation. The results

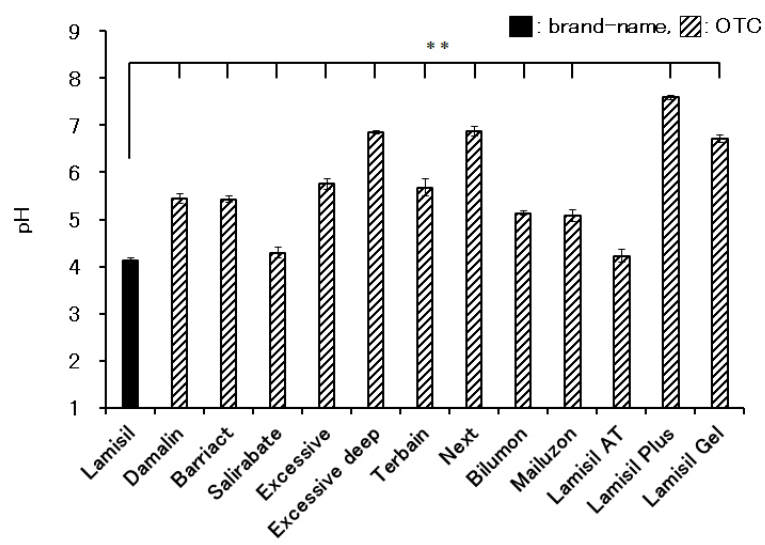

Figure 1. Measured pH of various preparations $(n=10)$. $*^{* *}<0.01$ (vs. LLamisil cream, Dunnett's test)

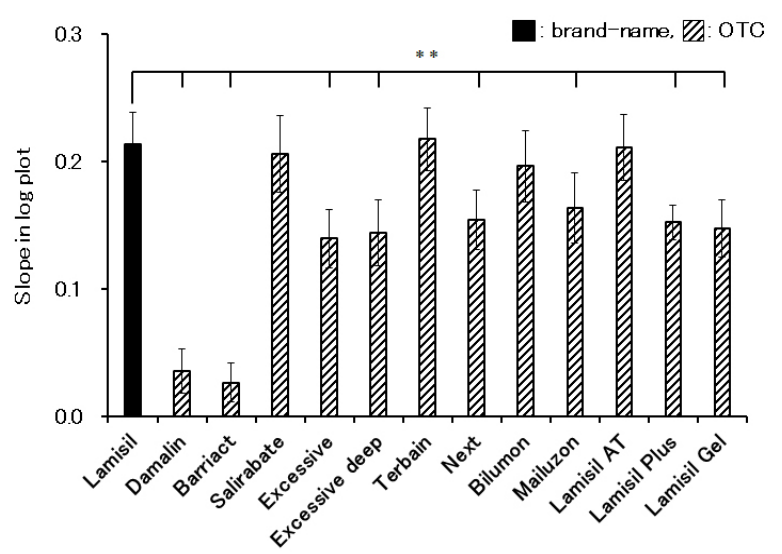

Figure 2. Measured slope for various creams $(n=8) .{ }^{* *} p<$ 0.01 (vs. Lamisil cream, Dunnett's test). are shown in Figures 2 and 3. The slope for Lamisil was 0.21 , whereas that for the OTC products ranged from 0.03 to 0.22 (Figure 2). In particular, the OTC products Damalin (0.04) and Barriact (0.03) had a significantly lower slope than that of Lamisil.

In contrast, the y-intercept for Lamisil was 3.11, whereas that for the OTC products ranged from 2.22 to 3.42, as shown in Figure 3. In particular, the OTC product Exiv Deep (2.22) had a significantly lower y-intercept than that of Lamisil, and Barriact (3.34) and Bilumon (3.42) had a higher $y$-intercept than that of Lamisil.

The yield value is known to be an index of cream ductility. As shown in Figure 4, the yield value for Lamisil was $128 \mathrm{dyn} / \mathrm{cm}^{2}$, whereas that for the OTC products ranged from 110 to $887 \mathrm{dyn} / \mathrm{cm}^{2}$. In particular, the OTC products Damalin $\left(887 \mathrm{dyn} / \mathrm{cm}^{2}\right)$, Barriact (512 dyn $/ \mathrm{cm}^{2}$ ), and Exiv Deep (663 dyn $/ \mathrm{cm}^{2}$ ) had a significantly higher yield value.

\subsection{Measurement of the squeeze force}

The squeeze force is expressed as the force required to expel a dose of each preparation. The squeeze force was measured by attaching a HapLog ${ }^{\circledR}$ to the right thumb

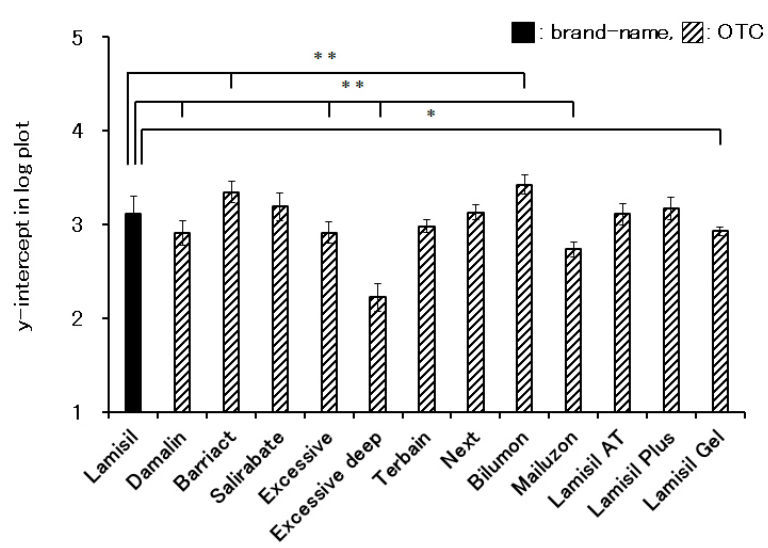

Figure 3. Y-intercept for various creams $(\boldsymbol{n}=\mathbf{8}) . * 0<0.05$, $*^{*} p<0.01$ (vs. Lamisil cream, Dunnett's test).

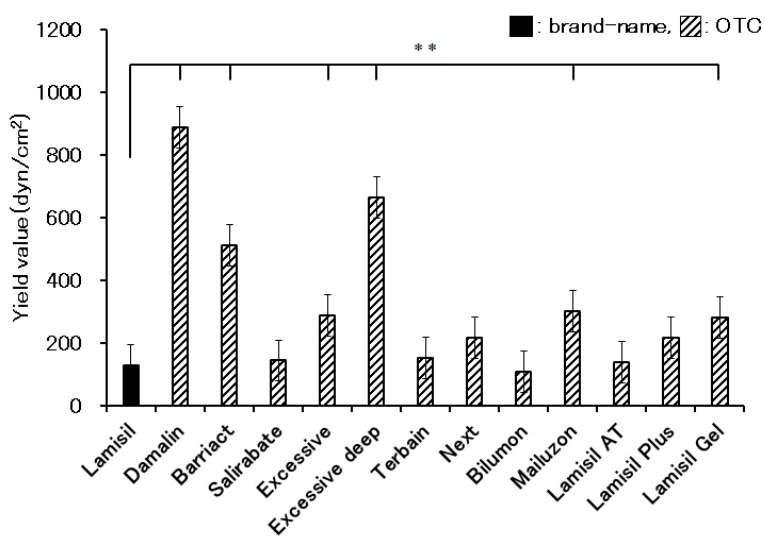

Figure 4. Yield value for various creams $(\boldsymbol{n}=\mathbf{8}) . * * p<0.01$ (vs. Lamisil cream, Dunnett's test). 


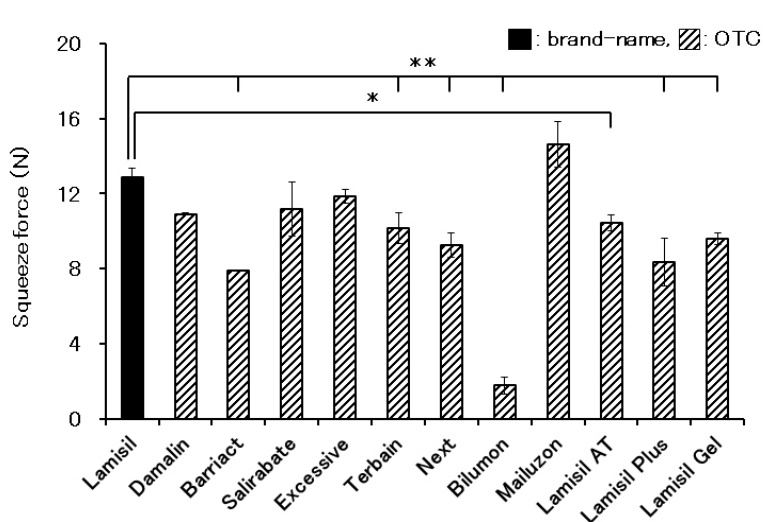

Figure 5. Squeeze force for various creams $(\boldsymbol{n}=\mathbf{3}){ }^{*} p<0.05$, $*^{*} p<0.01$ (vs. Lamisil cream, Dunnett's test).

and second finger, as shown in Figure 5. The squeeze force for Lamisil was $12.9 \mathrm{~N}$, whereas that for the OTC products ranged from 1.8 to $14.6 \mathrm{~N}$. In particular, Bilumon $(1.8 \mathrm{~N})$ had a significantly lower squeeze force. However, Exiv Deep was in a plastic bottle, so its squeeze force could not be measured because it was not in an aluminum or laminated tube.

\section{Discussion}

External preparations are expected to have both desirable pharmaceutical and pharmacological properties as well as other appropriate physiological and psychorheological characteristics such as tactile feel, ductility, color, and smell (19). Since patients rub ointments and creams onto the affected area by hand, poorly ductile and highly viscous preparations are considered difficult to apply, and a study has that suggested the need to improve ointment bases for patient convenience and comfort (20).

In general, external preparations with a $\mathrm{pH}$ close to that of the surface of skin reportedly have less skin corrosivity and cause less irritation. The $\mathrm{pH}$ of the skin is neutral near the epidermis, mildly acidic in the stratum corneum, and 4.5-6.0 on the surface (21). This suggests that a $\mathrm{pH}$ of 4.5-6.0, which is the same as the $\mathrm{pH}$ of the surface of the skin, is optimal for external preparations. Since the use of external preparations itself may cause exacerbation of symptoms in patients with sensitive skin, preparations with a $\mathrm{pH}$ close to that of the surface of the skin need to be selected to avoid that risk.

In this study, Exiv Deep had a significantly higher $\mathrm{pH}$ than the brand-name drug Lamisil, followed by Next, Lamisil Plus, and Lamisil Gel (Figure 1). Since Exiv Deep contains $10 \%$ urea, which has keratolytic and keratin peeling action, and Next, Lamisil Plus, and Lamisil Gel contain 2\% 1-menthol, which can cause irritation, in addition to the active ingredient, caution is required when selling these products to patients with sensitive skin depending on the condition of the affected area (Table 2).

A spreadmeter was used to determine the ductility

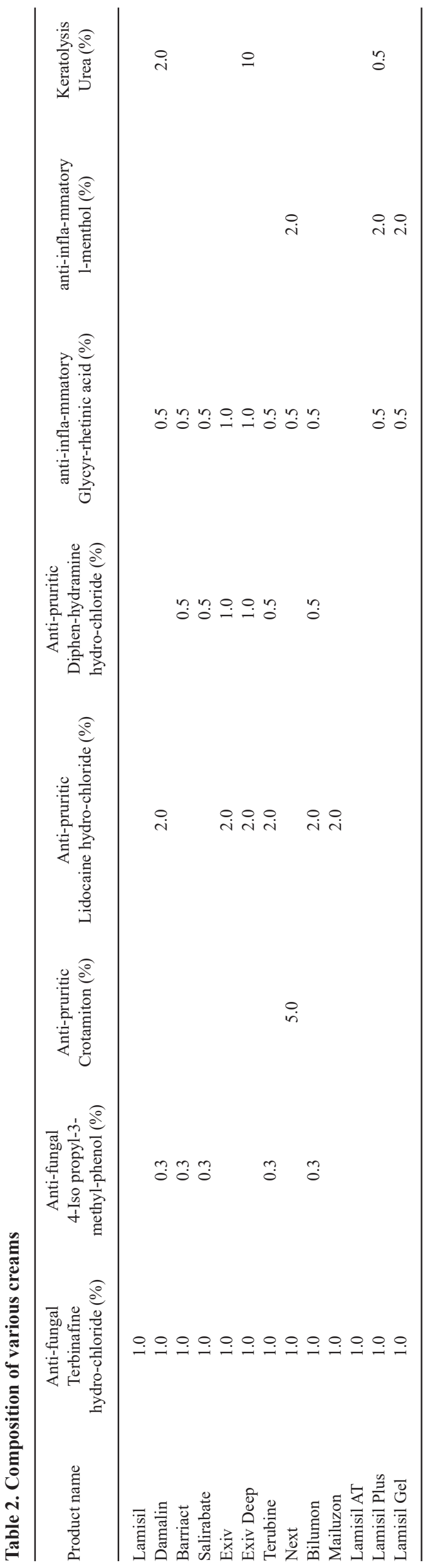


of various creams. A semilog graph was prepared with the diameter of the spread of the preparation $(\mathrm{cm})$ along the vertical axis and the time that the cream needed to spread (seconds) along the horizontal axis. A linear formula was derived for the log approximation curve for the plot. Based on the brand-name drug (Lamisil), a higher "slope" is considered to indicate greater ductility, and a higher "y-intercept" is considered to indicate lower viscosity (Figures 2 and 3). Damalin and Barriact had a significantly lower "slope," and they were deemed to be poorly "ductile" (Figure 2). Exiv Deep had a significantly lower "y-intercept" than that of the brand-name drug Lamisil, indicating it has a high "viscosity" (Figure 3).

The "yield value," which is the minimum stress that induces a semisolid such as a cream or ointment to deform or flow, is an index of the feel during use, and a preparation with a low "yield value" is considered to be easy to spread with minor force. When the "yield value" for the brand-name drug Lamisil and OTC products was compared, Damalin, Barriact, and Exiv Deep had a significantly higher "yield value." Damalin and Exiv Deep presumably had a low yield value partly because they contain urea at a respective concentration of 2 and $10 \%$ in addition to the active ingredient (Table 2). In contrast, Salirabate, Terbain, Bilumon, and Lamisil AT had a "yield value" close to that of the brand-name drug, and these preparations are considered to spread even with little force (Figure 4).

Measurement of the force of finger contact would presumably be possible while maintaining the free feeling of contact of the fingertip by measuring the force exerted on the fingertip directly in contact with the container using HapLog ${ }^{\circledR}$ attached to the fingers. With this method, a dose could be expelled with a "squeeze force" similar to, or lower than, that needed to expel Lamisil; this was true for the OTC products other than Mailuzon (Figure 5). A dose of Bilumon could be expelled with a squeeze force one-seventh that of Lamisil presumably because Bilumon is contained in a laminated tube while the other products are contained in aluminum tubes (Table 1) (16).

In conclusion, pharmacists need to recommend OTC products that cater to diverse patient needs based on the results of this study, but some OTC products contain a skin irritant (1-menthol) or skin-softening ingredients (Irish moss chondrus crispus, urea) and may exacerbate symptoms (Table 2). Since the properties of OTC products are not always appropriate for all patients, pharmacists need to properly select a preparation based on its physicochemical properties depending on the individual patient's skin condition.

\section{Acknowledgements}

The authors wish to thank Mr. Yusuke Gokan for providing technical assistance during part of the experimentation. The authors also wish to thank the
Frontier Science Business Division of Shiseido Co., Ltd., for supplying HapLog ${ }^{\circledR}$.

\section{References}

1. Ministry of Health, Labor, and Welfare. 2012 Overview of National Healthcare Costs. http://www.mhlw.go.jp/ toukei/saikin/hw/k-iryohi/12/dl/data.pdf (accessed August 6, 2018). (in Japanese)

2. Ministry of Health, Labor, and Welfare. Taxation of selfmedication (special exemption for deduction of medical expenses). http://www.mhlw.go.jp/stf/seisakunitsuite/ bunya/0000124853.html (accessed August 6, 2018). (in Japanese)

3. WHO. The Role of the Pharmacist in Self-Care and SelfMedication, (1998). http://apps.who.int/medicinedocs/ pdf/whozip32e/whozip32e.pdf (accessed August 6, 2018).

4. A reconstruction strategy for Japan - "Japan is Back," Cabinet decision on June 14, 2013. http://www.kantei. go.jp/jp/singi/keizaisaisei/pdf/saikou_jpn.pdf (accessed August 6, 2018). (in Japanese)

5. Wada Y, Nozawa M, Goto M, Shimokawa K, Ishii F. Generic selection criteria for safety and patient benefit [I]: Comparing the original drugs and generic ones in pharmaceutical properties. J Comm Pharm Sci. 2014; 6:97-105. (in Japanese)

6. Nozawa M, Wada Y, Yamazaki N, Shimokawa K, Ishii F. Generic selection criteria for safety and patient benefit [II]: Physicochemical characteristics of original and generic drugs for three different difluprednate-containing preparations (ointment, cream, and lotion). J Jpn Soc Comm Pharm. 2014; 2:37-48. (in Japanese)

7. Nozawa M, Goto M, Wada Y, Kumazawa M, Shimokawa K, Ishii F. Generic selection criteria for safety and patient benefit [VII]: Comparing the physicochemical and pharmaceutical properties of brand-name and generic terbinafine hydrochloride cream. Drug Discov Ther. 2018; 12:16-20.

8. Wada Y, Nozawa M, Goto M, Shimokawa K, Ishii F. Generic selection criteria for safety and patient benefit [III]: Comparing the pharmaceutical properties and patient usability of original and generic ophthalmic solutions containing timolol maleate. J Jpn Soc Pharm Health Sci. 2015; 41:394-403. (in Japanese)

9. Wada Y, Ami S, Nozawa M, Goto M, Shimokawa K, Ishii F. Generic selection criteria for safety and patient benefit [V]: Comparing the pharmaceutical properties and patient usability of original and generic nasal spray containing ketotifen fumarate. Drug Discov Ther. 2016; 10:88-92.

10. Wada Y, Kihara M, Nozawa M, Shimokawa K, Ishii F. Generic selection criteria for safety and patient benefit [IV]: Physicochemical and pharmaceutical properties of brand-name and generic ketoprofen tapes. Drug Discov Ther. 2015; 9:229-233.

11. Wada Y, Takaoka Y, Nozawa M, Goto M, Shimokawa K, Ishii F. Generic selection criteria for safety and patient benefit [VI]: Comparing the physicochemical and pharmaceutical properties of brand-name, generic, and OTC felbinac tapes. Drug Discov Ther. 2016; 10:300306.

12. Pharmaceutical and Medical Devices Agency (PMDA). http://www.pmda.go.jp/english/index.html (accessed August 6, 2018). 
13. Watabe $\mathrm{K}$, Nakamura $\mathrm{S}$, Shimamoto T, Uramatsu T, Kishi H, Uemura T, Niiike H. Preparation and evaluation of new metronidazole gel using hydroxypropyl methylcellulose. Jpn J Pharm Palliat Care Sci. 2009; 2:39-43.

14. Yoshida M. Extensibility of beesoften oil cream at various temperatures of $0.3 \%$. Jpn J Med Pharm Sci. 2014; 71:449-454. (in Japanese)

15. Komoda M, Akakuma S, Obara S, Koto A, Aono H, Kurahashi Y, Sugiyama N, Ishi N, Noda Y. Basic examination of bicarbonate sugar ointment neutralized reaction of leakage from gastrostomy fistula. Jpn Soc Par Ent Nutri. 2013; 28:819-825. (in Japanese)

16. Haptic Skill Logger, Kato Tech Co., Ltd. http://english. keskato.co.jp/ (accessed August 6, 2018).

17. Nakatani M, Kawasoe T. Haptic Skill Logger (HapLog): The wearable sensor for evaluating haptic behaviors. J Robo Soc Japan. 2012; 30:499-501. (in Japanese)
18. Yanai H. Excel Statistics in 4 Steps (4th Edition). OMS Publishing, Saitama, Japan, 2015. (in Japanese)

19. Shibata Y, Ikeda H, Kondou Y, Kihara K. Comparison of pharmaceutical properties of topical non-steroidal anti-inflammatory drug preparations on quality of life. Yakugaku Zasshi. 2005; 125:397-404. (in Japanese)

20. Nishizawa S, Yamaoka K, Yamaoka H, Yoshimura K, Hirabayashi S. Basic pharmaceutical research on formulation of kojic acid ointment for hospitals: In vitro release test and evaluation of permeability. Jpn J Pharm Health Care Sci. 2004; 30:20-26. (in Japanese)

21. Mizoguchi M, Ohara K, Aiba Y, Takado T, Hino H, Matsunaga K, Watanabe S. The pH of the skin. In: Encyclopedia of Skin. Asakura Bookstore, Tokyo, Japan, 2008; pp. 24. (in Japanese)

(Received July 27, 2018; Revised August 16, 2018; Accepted August 19, 2018) 